\title{
Editorial
}

\section{Special issue on "molecular targets, biomarkers and animal models for anti-cancer pharmacological research: potentials and challenges from chemoprevention to chemotherapeutic treatment"}

\author{
Ah-Ng Tony KONG ${ }^{1}$ \\ Center for Cancer Prevention Research, Ernest Mario School of Pharmacy, Rutgers, The State University of New Jersey, Piscataway, New \\ Jersey 08854, USA
}

\section{Key words}

cancer chemoprevention; dietary food factors; natural phytochemicals; molecular targets; biomarkers; animal models

${ }^{1}$ Correspondence to Prof Ah-Ng Tony KONG. Phn 1-732-444-3831 ext 228. Fax 1-732-445-3134. E-mail KongT@rci.rutgers.edu

Received 2007-07-25; Accepted 2007-07-25

doi: $10.1111 /$ j.1745-7254.2007.00699.x

With the current advances in the field of cancer research particularly in cancer chemoprevention, we have much to celebrate but we also have a lot of challenges before us. Some of the challenges include finding appropriate animal models for predicting efficacy of chemopreventive compounds for human cancers, of which are usually highly complex and involved multiple genes in nature and translating our basic research into clinical practice. One of the other challenges will be to disseminate our ideas into the larger biomedical and pharmaceutical community. With this in mind, I have been asked by the Editorial Team of Acta Pharmacologica Sinica to organize a special issue to discuss the current advances in cancer chemoprevention from prevention to treatment. The distinction and the potential overlapping nature of cancer chemoprevention versus chemotherapeutic treatment has become somewhat blurred, since prevention of carcinogenesis encompasses preventing any or all of the three stages of carcinogenesis namely initiation, promotion and progression. Oftentimes, many cancer chemopreventive compounds can possess all three biological properties that culminate in the overall protection against cancer. To be most effective, cancer chemoprevention of earlier lesions will be the ultimate goal if we are to eradicate this dreadful disease, since advanced metastasized cancers are almost lethal and unresponsive to radiation and chemotherapy. The reviews covered in this special issue, which are based on strong preclinical studies, will hopefully provide the impetus to translate this information to clinical practice and improved patient care. 